\title{
Altered intrinsic brain activities in patients with acute eye pain using amplitude of low-frequency fluctuation: a resting-state fMRI study
}

This article was published in the following Dove Press journal:

Neuropsychiatric Disease and Treatment

\author{
Zhi-Ming Pan' \\ Hai-Jun Li' \\ Jing $\mathrm{Bao}^{\prime}$ \\ Nan Jiang' \\ Qing Yuan' \\ Shelby Freeberg ${ }^{2}$ \\ Pei-Wen Zhu' \\ Lei Yel \\ Ming-Yang $\mathrm{Ma}^{\prime}$ \\ Xin Huang' \\ Yi Shao'
}

'Department of Ophthalmology and Radiology, The First Affiliated Hospital of Nanchang University, Nanchang, Jiangxi, People's Republic of China; ${ }^{2}$ Department of Ophthalmology, University of Florida, Gainesville, FL, USA
Correspondence: Yi Shao; Xin Huang Department of Ophthalmology, The First Affiliated Hospital of Nanchang University, No 17, Yongwaizheng Street, Donghu District, Nanchang 330006, Jiangxi, People's Republic of China $\mathrm{Tel}+8679188692520$ Email freebee99@।63.com; 334966891@qq.com
Objective: Many previous studies have reported that pain symptoms can lead to significant brain function and anatomical changes, whereas the intrinsic brain activity changes in acute eye pain (EP) patients remain unknown. Using the amplitude of low-frequency fluctuation (ALFF) method, this study aimed to evaluate the spontaneous brain activity alterations and their relationships with clinical features in acute EP patients.

Participants and methods: A total of 20 patients with EP (15 males and 5 females) and 20 healthy controls (HCs; 15 males and 5 females) closely matched in age, sex, and education underwent resting-state functional magnetic resonance imaging scans. The ALFF method was applied to assess spontaneous brain activity changes. The ALFF values of the EP patients were distinguished from those of the HCs using a receiver operating characteristic curve. Pearson's correlation analysis was used to investigate the relationships between the mean ALFF signal values from many brain regions and the clinical features in EP patients.

Results: Compared with the HCs, acute EP patients had significantly lower ALFF in the left and right precentral/postcentral gyrus and left precuneus. In contrast, acute EP patients showed higher ALFF values in the right and left parahippocampal gyri and left caudate. However, no relationship was observed between the mean ALFF signal values from the different areas and clinical manifestations in the acute EP patients.

Conclusion: We demonstrated that acute EP patients showed abnormal intrinsic brain activities in the precentral/postcentral gyrus and limbic system, which might provide useful information for explaining neural mechanisms in EP patients.

Keywords: eye pain, ALFF, blood oxygenation level dependent, resting state, functional magnetic resonance imaging

\section{Introduction}

Eye pain (EP) is a common symptom of eye disease. The cornea is supplied by the ophthalmic branch of the trigeminal nerve and is one of the most densely innervated structures in the body. Keratitis or a corneal ulcer will lead to severe ocular pain. According to a survey in Central China, the prevalence of infectious keratitis was $0.148 \%{ }^{1}$ At present, antibiotics are the main treatment for keratitis or corneal ulcers. ${ }^{2}$ However, there have been no effective measures to alleviate keratitis-associated ocular pain. The direct cause of corneal pain occurs in cases in which corneal nerve damage triggers the peripheral and central trigeminal sensory network; ${ }^{3}$ this process is associated with cluster headaches. ${ }^{4}$ Meanwhile, EP is a symptom of dry eye, and many previous studies have demonstrated that dry eye patients have accompanying psychological disorders such as anxiety and depressive symptoms as a result of chronic EP.5,6 
Functional magnetic resonance imaging (fMRI) has been successfully applied to evaluate brain activity changes in many pain-related diseases. A previous study demonstrated that migraineurs showed pain- and cognitive-function-altered amplitude of low-frequency fluctuation (ALFF) values in many brain regions. ${ }^{7}$ Flodin et $\mathrm{al}^{8}$ reported that patients with pain due to chronic rheumatoid arthritis showed increased brain connectivity in the supplementary motor areas, midcingulate cortex, and primary sensorimotor cortex. Meanwhile, tension-type headache patients showed lower synchronization of neuronal activity in many pain-associated brain regions. ${ }^{9}$ Zhang et $\mathrm{al}^{10}$ demonstrated that acute low-back pain was associated with a decrease in ALFF in the right posterior cingulate cortex/precuneus and left primary somatosensory cortex (S1). The previously described studies confirm that pain symptoms can cause brain activity changes. However, there have been few studies on brain activity changes in EP patients, and it remains unknown whether acute EP leads to brain activity changes. ALFF, an fMRI method, can be used to detect the spontaneous neuronal activity in bloodoxygenation-level-dependent (BOLD)-related signals. In addition to spontaneous low-frequency fluctuations in BOLD, fMRI signals also correlated with the spontaneous neuronal activities. ${ }^{11,12}$ In our previous studies, the ALFF method was successfully applied to the investigation of spontaneous brain activities in various diseases such as glaucoma and blindness. ${ }^{13,14}$ Thus, the ALFF method was proven to be a reliable and effective resting-state fMRI technique.

Using the ALFF method, the aim of this study was to investigate different intrinsic brain activities in acute EP patients compared to healthy controls (HCs) and correlate these results with clinical manifestations in the patients.

\section{Participants and methods Participants}

A total of 20 acute EP patients (15 males and 5 females) were recruited from the Ophthalmology Department of the First Affiliated Hospital of Nanchang University. The diagnostic criteria for EP patients consisted of two main points: 1) all acute EP patients with keratitis or corneal ulcer of the right eye and 2) bilateral eyes without any other ocular diseases (strabismus, amblyopia, cataracts, glaucoma, optic neuritis, retinal degeneration, or others).

The exclusion criteria for EP patients in this study consisted of several points: 1) chronic EP symptoms for a long time; 2) EP due to ocular trauma or glaucoma; 3) EP with serious related complications (ocular perforation, endophthalmitis, orbital cellulitis, or other serious conditions); 4) EP patients with histories of painkiller treatment; 5) EP patients with associated pain such as headache or pain in other parts of the body; 6) cardiovascular diseases such as heart disease or hypertension; 7) psychiatric disorders; and 8) cerebral infarction diseases (cerebral hemorrhage, cerebral infarction, or cerebral vascular malformations).

Twenty HCs (15 males and 5 females) with age-, sex-, and education-status-matched subjects in the EP group participated in the study. All of the HCs met the following criteria: 1) no psychiatric disorders (depression, bipolar disorder, or other psychiatric disorders) and 2) being able to undergo MRI (no cardiac pacemaker or implanted metal devices).

The protocol followed the Declaration of Helsinki and was approved by The Human Research Ethics Committee of the First Affiliated Hospital of Nanchang University. All the volunteers participated voluntarily and were informed of the purposes, methods, and potential risks before signing the informed consent.

\section{MRI parameters}

MRI scanning was performed on a 3 Tesla MRI scanner (Trio, Siemens, Munich, Germany). fMRI data were obtained using a spoiled gradient-recalled echo sequence with the following parameters: number of structure images $=176$, repetition time $=1.900 \mathrm{~ms}$, echo time $=2.26 \mathrm{~ms}$, thickness $=1.0 \mathrm{~mm}$, gap $=0.5 \mathrm{~mm}$, acquisition matrix $=256 \times 256$, field of view $=250 \times 250 \mathrm{~mm}$, and flip angle $=9^{\circ}$. Finally, 240 functional images (repetition time $=2,000 \mathrm{~ms}$, echo time $=30 \mathrm{~ms}$, thickness $=4.0 \mathrm{~mm}$, gap $=1.2 \mathrm{~mm}$, acquisition matrix $=64 \times 64$, flip angle $=90^{\circ}$, field of view $=220 \times 220 \mathrm{~mm}$, and 29 axial slices with gradient-recalled echo-planar imaging pulse sequence) were obtained.

\section{fMRI data analysis}

Functional data were preprocessed using DPARSFA (http:// rfmri.org/DPARSF) ${ }^{15}$ with the Statistical Parametric Mapping software (SPM8, http://www.fil.ion.ucl.ac.uk/spm) implemented in MATLAB 2013a (MathWorks, Natick, MA, USA) and included several steps: 1) the first ten volumes from each subject were discarded due to the signal reaching equilibrium and the participants' adaptation to the scanning noise; 2) after head motion correction, the motion time courses were obtained by estimating the values for translation $(\mathrm{mm})$ and rotation (degrees) for each subject. The data for participants who had more than $1.5 \mathrm{~mm}$ maximum displacement in the $x$, $y$, or $z$ axes or $1.5^{\circ}$ of angular motion during the entire fMRI scan would be rejected; 3 ) the nuisance covariate effects of nonneuronal BOLD fluctuations, including white matter and cerebrospinal fluid signals, were also removed by a linear regression process; and 4) fMRI images were spatially 
normalized to the Montreal Neurological Institute space criteria using the standard echo-planar imaging template and resampling the images at a resolution of $3 \times 3 \times 3 \mathrm{~mm}^{3}$.

\section{ALFF analysis}

First, to calculate ALFF, the remaining data were smoothed with a Gaussian kernel of $6 \times 6 \times 6 \mathrm{~mm}^{3}$ full width at halfmaximum. Second, the fMRI images were detrended and bandpass-filtered $(0.01-0.08 \mathrm{~Hz})$ to reduce the effects of low-frequency drift and physiological high-frequency respiratory and cardiac noise. We then converted the smoothed signal of each voxel from time domain to frequency domain via a fast Fourier transform algorithm to obtain the power spectrum. Finally, all of the ALFF maps were divided by the mean value of each ALFF map. ${ }^{16}$

\section{Statistical analysis}

The cumulative clinical measurements, including the duration of the onset of EP, were analyzed in this study with an independent sample $t$-test using SPSS version 16.0 (SPSS Inc, Chicago, IL, USA), where $p<0.05$ was considered to represent a significant difference.

A two-sample $t$-test was used to examine the difference in the z ALFF maps between the EP groups and the HC using the SPM8 toolkit after controlling for the effects of age and sex $(p<0.01$ for multiple comparisons using Gaussian random-field theory $(z>2.3, p<0.01$, cluster $>40$ voxels, FDR corrected)).

The mean ALFF values in the different brain regions between the two groups were analyzed using the receiver operating characteristic (ROC) curve method. Pearson's correlation was used to evaluate the relationship between the mean ALFF values in different brain regions in the EP group, where $p<0.05$ was considered to represent a significant difference.

\section{Results}

\section{Demographics and visual measurements}

We did not find any differences in weight $(p=0.732)$ or age ( $p=0.933)$ between the two groups $(p>0.05)$. The mean \pm standard deviation of acute EP duration was $6.80 \pm 2.12$ days (more details are listed in Table 1).

\section{ALFF differences}

Compared with the HCs, the acute EP patients had significantly lower ALFF values in the left and right precentral/postcentral gyri and left precuneus (Figure $1 \mathrm{~A}$ and $\mathrm{B}$ [blue] and Table 2). Meanwhile, higher ALFF values in the EP patient groups were observed in the right and left parahippocampa
Table I Demographics and clinical measurements by group

\begin{tabular}{lllll}
\hline Condition & EP & HC & $\boldsymbol{t}$ & p-value* \\
\hline Male/female & $15 / 5$ & $\mathrm{I} / 5$ & $\mathrm{~N} / \mathrm{A}$ & $>0.99$ \\
Age (years) & $37.85 \pm 5.63$ & $38.00 \pm 5.62$ & -0.084 & 0.933 \\
Weight (kg) & $69.30 \pm 6.04$ & $69.95 \pm 5.89$ & -0.344 & 0.732 \\
Handedness & $20 \mathrm{R}$ & $20 \mathrm{R}$ & $\mathrm{N} / \mathrm{A}$ & $>0.99$ \\
Duration of EP (days) & $6.80 \pm 2.12$ & $\mathrm{~N} / \mathrm{A}$ & $\mathrm{N} / \mathrm{A}$ & $\mathrm{N} / \mathrm{A}$ \\
\hline
\end{tabular}

Notes: $* p<0.05$. Independent $t$-tests comparing two groups.

Abbreviations: EP, eye pain; $\mathrm{HC}$, healthy control; N/A, not applicable.

gyri and left caudate (Figure 1A and B [red] and Table 2; $p<0.01$ for multiple comparisons using the Gaussian random-field theory, $z>2.3, p<0.01$, cluster $>40$ voxels, FDR corrected). The mean values of altered ALFF values between the two groups are represented using a histogram (Figure 1C). In the EP group, we did not find any correlation between the mean ALFF values in different brain regions and their clinical manifestations $(p>0.05)$.

\section{ROC curve}

The mean ALFF values in the different brain regions were analyzed using the ROC curve method. The area under the ROC curve indicated diagnosis rate. The areas under the ROC curve for ALFF values were as follows: left precentral/ postcentral gyrus $(0.827, p<0.001 ; 95 \%$ confidence interval [CI]: 0.696-0.959), right precentral/postcentral gyrus (0.873, $p<0.001 ; 95 \%$ CI: $0.766-0.979)$, left precuneus (0.847, $p<0.001 ; 95 \% \mathrm{CI}: 0.725-0.970$; Figure 2A, EPs $<$ HCs $)$, right parahippocampal gyrus $(0.905, p<0.001$; 95\% CI: 0.815-0.995), left parahippocampal gyrus $(0.910 ; p<0.001 ; 95 \%$ CI: $0.820-1.000)$, and left caudate $(0.947, p<0.001 ; 95 \%$ CI: $0.884-1.000$; Figure 2B, EPs $>$ HCs).

\section{Discussion}

To the best of our knowledge, this study is the first to investigate intrinsic brain activity changes in acute EP patients using the ALFF method. Compared with the HCs, the acute EP patients showed significantly lower ALFF values in the left and right precentral/postcentral gyri and left precuneus. In contrast, the acute EP patients showed higher ALFF values in the right and left parahippocampal gyri and left caudate. However, no relationship was found between the mean ALFF signal values of the different areas and the clinical manifestations in the acute EP patients.

\section{Analysis of decreased ALFF values in the EP group}

The postcentral gyrus is located in the primary somatosensory cortex (S1), which plays a critical role in sensory function. ${ }^{17}$ 


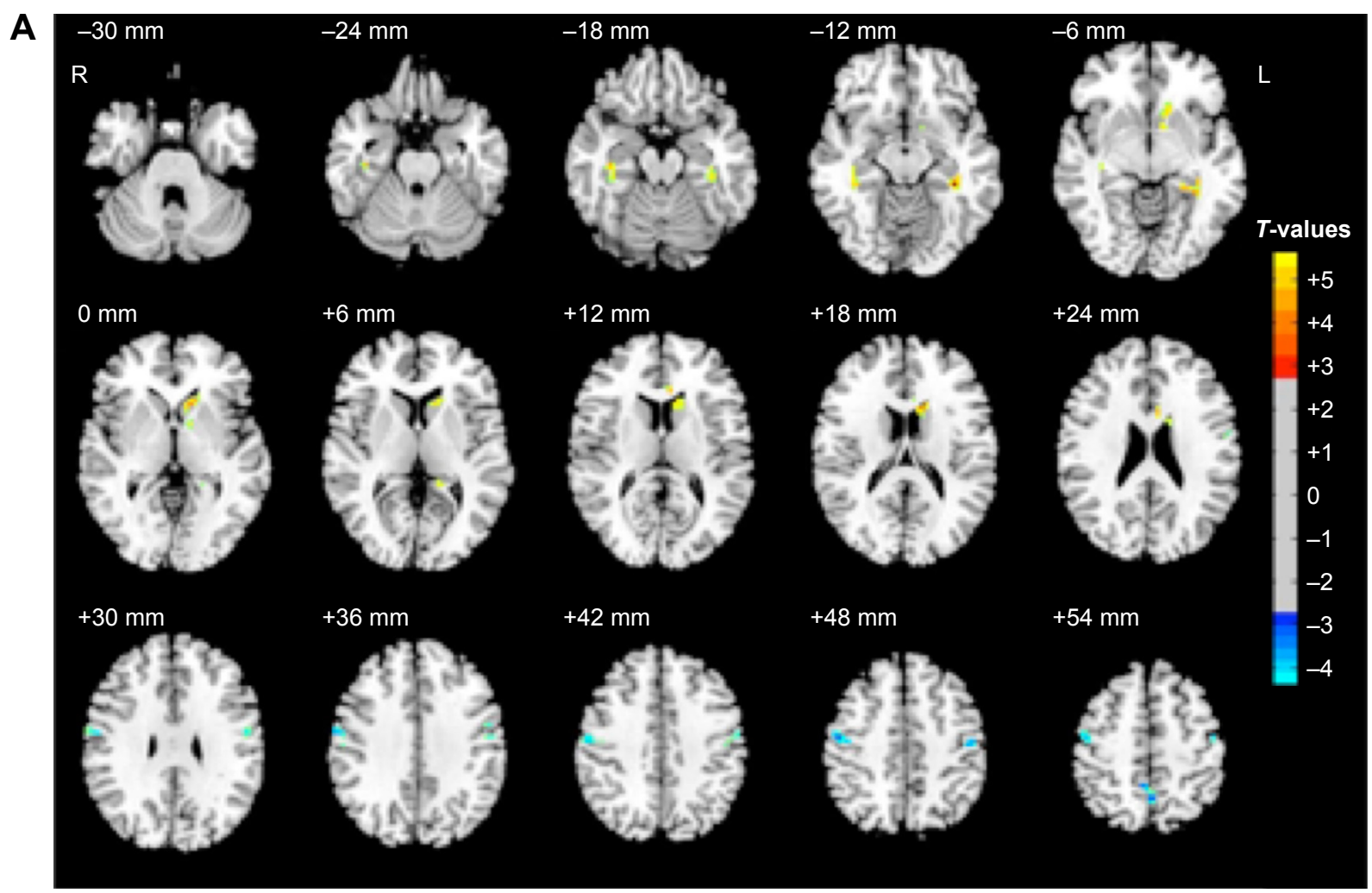

B L
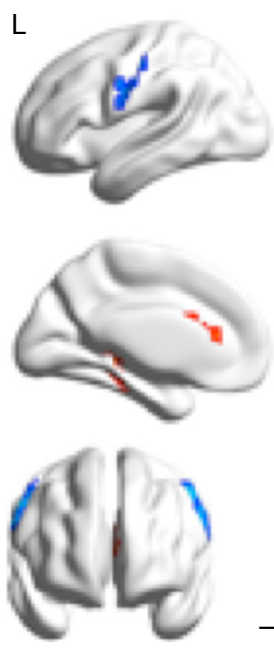
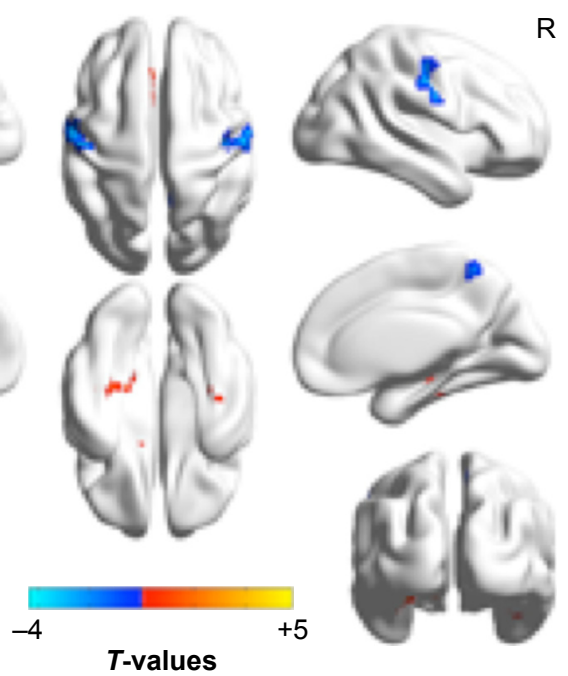

C

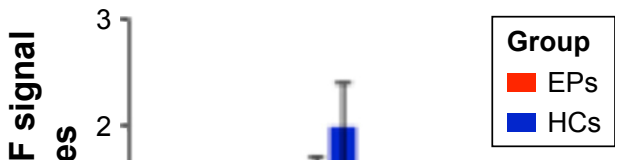

$\mathrm{C}$

I Spontaneous brain activity in the EPs versus HCs.

Notes: (A and B) Significant activity differences were observed in the LPreCG/PosCG, RPreCG/PosCG, LPrecu, RParaHG, LParaHG, and left caudate. The red or yellow denotes higher ALFF values, and the blue areas indicate lower ALFF values, respectively $(p<0.01$ for multiple comparisons using Gaussian-random field theory, $z>2.3$, $p<0.0$ I, cluster $>40$ voxels, FDR corrected). (C) The mean values of altered ALFF values between the EPs and HC groups.

Abbreviations: ALFF, amplitude of low-frequency fluctuation; EP, eye pain; FDR, false discovery rate; HC, healthy control; LParaHG, left parahippocampal gyrus; LPreCG/ PosCG, left precentral/postcentral gyrus; LPrecu, left precuneus; RParaHG, right parahippocampal gyrus; RPreCG/PosCG, right precentral/postcentral gyrus.

The $\mathrm{S} 1$ is also involved in pain coding. ${ }^{18,19}$ Wrigley et $\mathrm{al}^{20}$ demonstrated that spinal cord injury with neuropathic pain was associated with $\mathrm{S} 1$ reorganization. Meanwhile, chronic back pain patients were reported to exhibit accompanying $\mathrm{S} 1$ reorganization. ${ }^{21}$ A recent study demonstrated that $\mathrm{S} 1$ sensitivity was related to limb pain. ${ }^{22}$ Migraine patients showed a decreased cerebral blood flow in the $\mathrm{S} 1 .{ }^{23}$
Moreover, Moulton et $\mathrm{al}^{24}$ found that corneal pain with blinking showed S1 and primary motor cortex activation. Consistent with these findings, we demonstrated that acute EP patients showed significant decreases in ALFF values in the bilateral precentral/postcentral gyrus (BA 3, 4), which indicate decreased $\mathrm{S} 1$ brain activities. Thus, we speculated that the acute EP might lead to the dysfunction of the S1. 
Table 2 Brain areas with significantly different ALFF values between groups

\begin{tabular}{|c|c|c|c|c|c|c|}
\hline \multirow[t]{2}{*}{$\begin{array}{l}\text { Brain regions/ } \\
\text { conditions }\end{array}$} & \multirow[t]{2}{*}{ BA } & \multicolumn{3}{|c|}{$\begin{array}{l}\text { MNI } \\
\text { coordinates }\end{array}$} & \multirow[t]{2}{*}{$\begin{array}{l}\text { Cluster } \\
\text { size }\end{array}$} & \multirow[t]{2}{*}{$t$-value } \\
\hline & & $x$ & $\mathbf{Y}$ & $\mathbf{Z}$ & & \\
\hline \multicolumn{7}{|l|}{$\mathrm{EP}<\mathrm{HCs}$} \\
\hline $\begin{array}{l}\text { Left precentral/ } \\
\text { postcentral gyrus }\end{array}$ & $3 / 4$ & -48 & -18 & 48 & 53 & -3.593 \\
\hline $\begin{array}{l}\text { Right precentral/ } \\
\text { postcentral gyrus }\end{array}$ & $3 / 4$ & 51 & -12 & 48 & 80 & -4.074 \\
\hline Left precuneus & 7 & 3 & -51 & 57 & 54 & -4.374 \\
\hline \multicolumn{7}{|l|}{$\mathrm{EP}>\mathrm{HCs}$} \\
\hline $\begin{array}{l}\text { Right parahippocampal } \\
\text { gyrus }\end{array}$ & 36 & 39 & -21 & -21 & 41 & 5.002 \\
\hline $\begin{array}{l}\text { Left parahippocampal } \\
\text { gyrus }\end{array}$ & 36 & -36 & -36 & -12 & 63 & 5.803 \\
\hline Left caudate & - & -12 & 18 & -3 & 112 & 5.154 \\
\hline
\end{tabular}

Note: The statistical threshold was set at the voxel level with $p<0.05$ for multiple comparisons using Gaussian random-field theory $(z>2.3, p<0.0$ l, cluster $>40$ voxels, FDR corrected).

Abbreviations: ALFF, amplitude of low-frequency fluctuation; BA, Brodmann area; EP, eye pain; FDR, false discovery rate; HC, healthy control; L, left; MNI, Montrea Neurological Institute; R, right.

The precuneus (BA7) is a part of the superior parietal lobule, which plays a critical role in coordination of motor and working memory. ${ }^{25,26}$ The precuneus is a core part of the default mode network (DMN). ${ }^{27}$ Meanwhile, the precuneus was shown to be related to pain sensitivity in healthy adults. ${ }^{28}$ A disturbed DMN has been found for a variety of pain-related diseases such as migraine headaches, dysmenorrhea, and back pain. ${ }^{29-31}$ In our study, we demonstrated that the acute EP patients showed decreased ALFF values in the left precuneus, which indicated left precuneus dysfunction. We speculated that acute EP might lead to DMN dysfunction.

\section{Analysis of increased ALFF values in the EP group}

The parahippocampal gyrus plays an important role in memory, ${ }^{32,33}$ especially visual memory. ${ }^{34}$ In addition, it is a core part of the limbic system, which plays a critical role in memory and emotions. ${ }^{35}$ Zhang et $\mathrm{al}^{10}$ demonstrated that back pain patients showed altered regional homogeneity values in the parahippocampal gyrus. Various pain-related diseases have also been associated with activation of the limbic system..$^{36,37}$ Meanwhile, the parahippocampal gyrus has been shown to be related to negative emotions, and Chan et al ${ }^{38}$ reported that negative emotions correlated with parahippocampal gyrus activity. A previous study demonstrated that the pain symptoms were closely related to negative emotions. ${ }^{39,40}$ In this study, we found that EP patients showed an increase in ALFF values in the bilateral parahippocampal gyrus, which might reflect hyperactivity in the limbic system. Moreover, parahippocampal gyrus activation might reflect negative emotions that occur in EP patients. The caudate is a part of the basal ganglia, which is involved in motor tasks
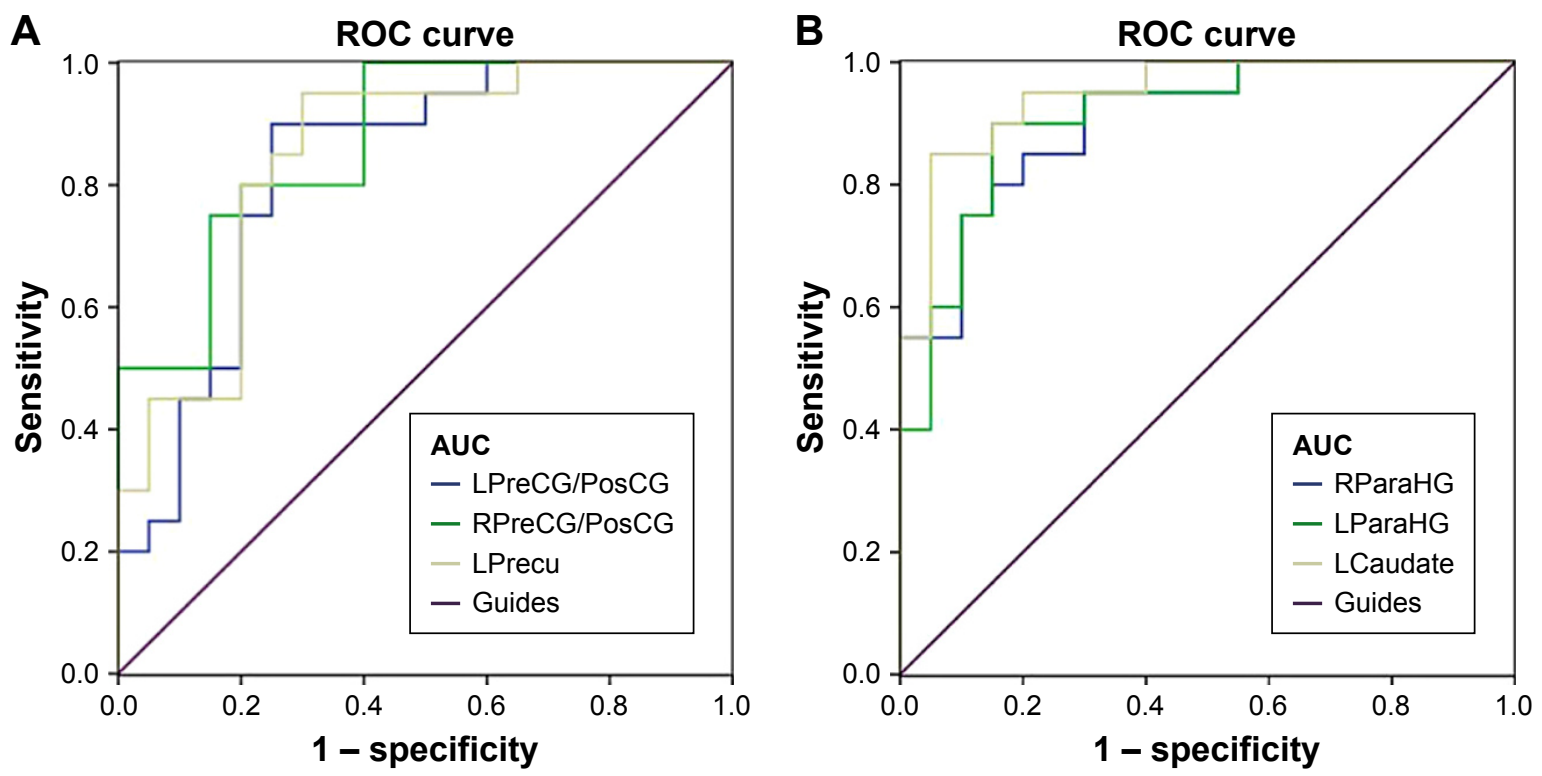

Figure 2 ROC curve analysis of the mean ALFF values for altered brain regions.

Notes: $(\mathbf{A})$ The areas under the ROC curve were 0.827 ( $p<0.001$; $95 \%$ Cl: 0.696-0.959) for LPreCG/PosCG; RPreCG/PosCG (0.873) ( $p<0.00$ I; $95 \%$ Cl: $0.766-0.979)$; LPrecu (0.847) ( $p<0.00$ I; 95\% Cl: 0.725-0.970), (EPs < HCs). (B) RParaHG (0.905) ( $p<0.00$ I; 95\% Cl: 0.8I5-0.995) and LParaHG (0.9I0) ( $p<0.00 \mathrm{I} ; 95 \%$ Cl: 0.820-I.000); left caudate (0.947) ( $p<0.00$ I; 95\% Cl: 0.884-I.000) (EPs $>$ HCs).

Abbreviations: ALFF, amplitude of low-frequency fluctuation; Cl, confidence interval; EP, eye pain; HC, healthy control; LParaHG, left parahippocampal gyrus; LPreCG/ PosCG, left precentral/postcentral gyrus; LPrecu, left precuneus; RParaHG, right parahippocampal gyrus; RPreCG/PosCG, right precentral/postcentral gyrus; ROC, receiver operating characteristic. 
and cognitive function. ${ }^{41-43}$ In addition, the caudate nucleus plays an important role in pain modulation. ${ }^{44,45}$ In our study, we found that acute EP patients showed higher ALFF values in the left caudate, which indicates caudate hyperfunction.

\section{Conclusion}

In summary, we have demonstrated that acute EP patients show intrinsic brain activity alterations in $\mathrm{S} 1$ regions and the limbic system, which might provide useful information for explaining neural mechanisms in EP patients. The ALFF values might be useful as a clinical marker to reflect pain severity in acute EP patients.

\section{Acknowledgment}

This study was supported by the National Natural Science Foundation of China (No: 81660158, 81160118, 81460092, 81400372).

\section{Disclosure}

The authors report no conflicts of interest in this work.

\section{References}

1. Cao J, Yang Y, Yang W, et al. Prevalence of infectious keratitis in Central China. BMC Ophthalmol. 2014;14:43.

2. Lalitha P, Manoharan G, Karpagam R, et al. Trends in antibiotic resistance in bacterial keratitis isolates from South India. Br J Ophthalmol. 2017;101(2):108-113.

3. Rosenthal P, Borsook D. Ocular neuropathic pain. Br J Ophthalmol. 2016;100(1):128-134.

4. Sandrini G, Alfonsi E, Ruiz L, et al. Impairment of corneal pain perception in cluster headache. Pain. 1991;47(3):299-304.

5. Szakáts I, Sebestyén M, Németh J, Birkas E, Purebl G. The role of health anxiety and depressive symptoms in dry eye disease. Curr Eye Res. 2016;41(8):1044-1049.

6. Zhang Y, Lin T, Jiang A, Zhao N, Gong L. Vision-related quality of life and psychological status in Chinese women with Sjogren's syndrome dry eye: a case-control study. BMC Womens Health. 2016;16(1):75.

7. Wang JJ, Chen X, Sah SK, et al. Amplitude of low-frequency fluctuation (ALFF) and fractional ALFF in migraine patients: a resting-state functional MRI study. Clin Radiol. 2016;71(6):558-564.

8. Flodin P, Martinsen S, Altawil R, et al. Intrinsic brain connectivity in chronic pain: a resting-state fMRI study in patients with rheumatoid arthritis. Front Hum Neurosci. 2016;10:107.

9. Wang P, Du H, Chen N, et al. Regional homogeneity abnormalities in patients with tension-type headache: a resting-state fMRI study. Neurosci Bull. 2014;30(6):949-955.

10. Zhang SS, Wu W, Yang JM, Wang CH. Abnormal spontaneous brain activity in acute low-back pain revealed by resting-state functional MRI. Am J Phys Med Rehabil. 2017;96(4):253-259.

11. Goldman RI, Stern JM, Engel J Jr, Cohen MS. Simultaneous EEG and fMRI of the alpha rhythm. Neuroreport. 2002;13(18):2487-2492.

12. Logothetis NK, Pauls J, Augath M, Trinath T, Oeltermann A. Neurophysiological investigation of the basis of the fMRI signal. Nature. 2001;412:150-157.

13. Huang X, Zhong YL, Zeng XJ, et al. Disturbed spontaneous brain activity pattern in patients with primary angle-closure glaucoma using amplitude of low-frequency fluctuation: a fMRI study. Neuropsychiatr Dis Treat. 2015;11:1877-1883.
14. Li Q, Huang X, Ye L, et al. Altered spontaneous brain activity pattern in patients with late monocular blindness in middle-age using amplitude of low-frequency fluctuation: a resting-state functional MRI study. Clin Interv Aging. 2016;11:1773-1780.

15. Chao-Gan Y, Yu-Feng Z. DPARSF: A MATLAB toolbox for "pipeline" data analysis of resting-state fMRI. Front Syst Neurosci. 2010; 14(4):13.

16. Zang YF, He Y, Zhu CZ, et al. Altered baseline brain activity in children with ADHD revealed by resting-state functional MRI. Brain Dev. 2007;29(2):83-91.

17. Kaukoranta E, Hämäläinen M, Sarvas J, Hari R. Mixed and sensory nerve stimulations activate different cytoarchitectonic areas in the human primary somatosensory cortex SI. Neuromagnetic recordings and statistical considerations. Exp Brain Res. 1986;63(1):60-66.

18. Timmermann L, Ploner M, Haucke K, Schmitz F, Baltissen R, Schnitzler A. Differential coding of pain intensity in the human primary and secondary somatosensory cortex. J Neurophysiol. 2001;86(3): 1499-1503.

19. Canavero S, Bonicalzi V. Role of primary somatosensory cortex in the coding of pain. Pain. 2013;154(3):334-344.

20. Wrigley PJ, Press SR, Gustin SM, et al. Neuropathic pain and primary somatosensory cortex reorganization following spinal cord injury. Pain. 2009;141(1-2):52-59.

21. Flor H, Braun C, Elbert T, Birbaumer N. Extensive reorganization of primary somatosensory cortex in chronic back pain patients. Neurosci Lett. 1997;224(1):5-8.

22. Zhao J, Guo X, Xia X, et al. Functional reorganization of the primary somatosensory cortex of a phantom limb pain patient. Pain Physician. 2016;19(5):E781-E786.

23. Hodkinson DJ, Veggeberg R, Wilcox SL, et al. Primary somatosensory cortices contain altered patterns of regional cerebral blood flow in the interictal phase of migraine. PLoS One. 2015;10(9):e0137971.

24. Moulton EA, Becerra L, Rosenthal P, Borsook D. An approach to localizing corneal pain representation in human primary somatosensory cortex. PLoS One. 2012;7(9):e44643.

25. Wenderoth N, Debaere F, Sunaert S, Swinnen SP. The role of anterior cingulate cortex and precuneus in the coordination of motor behaviour. Eur J Neurosci. 2005;22(1):235-246.

26. Wallentin M, Weed E, Østergaard L, Mouridsen K, Roepstorff A. Accessing the mental space-Spatial working memory processes for language and vision overlap in precuneus. Hum Brain Map. 2008;29(5):524-532.

27. Utevsky AV, Smith DV, Huettel SA. Precuneus is a functional core of the default-mode network. J Neurosci. 2014;34(3):932-940.

28. Goffaux P, Girard-Tremblay L, Marchand S, Daigle K, Whittingstall K. Individual differences in pain sensitivity vary as a function of precuneus reactivity. Brain Topogr. 2014;27(3):366-374.

29. Zhang J, Su J, Wang M, et al. Increased default mode network connectivity and increased regional homogeneity in migraineurs without aura. J Headache Pain. 2016;17(1):98.

30. Wu TH, Tu CH, Chao HT, et al. Dynamic changes of functional pain connectome in women with primary dysmenorrhea. Sci Rep. 2016;6: 24543.

31. Letzen JE, Robinson ME. Negative mood influences default mode network functional connectivity in patients with chronic low back pain: implications for functional neuroimaging biomarkers. Pain. 2017;158(1): 48-57.

32. Yonelinas AP, Hopfinger JB, Buonocore MH, Kroll NE, Baynes K. Hippocampal, parahippocampal and occipital-temporal contributions to associative and item recognition memory: an fMRI study. Neuroreport. 2001;12(2):359-363.

33. Kilpatrick L, Cahill L. Amygdala modulation of parahippocampal and frontal regions during emotionally influenced memory storage. Neuroimage. 2003;20(4):2091-2099.

34. Düzel E, Habib R, Rotte M, Guderian S, Tulving E, Heinze HJ. Human hippocampal and parahippocampal activity during visual associative recognition memory for spatial and nonspatial stimulus configurations. J Neurosci. 2003;23(28):9439-9444. 
35. Catani M, Dell'acqua F, Thiebaut de Schotten M. A revised limbic system model for memory, emotion and behaviour. Neurosci Biobehav Rev. 2013;37(8):1724-1737.

36. Younger JW, Shen YF, Goddard G, Mackey SC. Chronic myofascial temporomandibular pain is associated with neural abnormalities in the trigeminal and limbic systems. Pain. 2010;149(2):222-228.

37. Fenton BW. Limbic associated pelvic pain: a hypothesis to explain the diagnostic relationships and features of patients with chronic pelvic pain. Med Hypotheses. 2007;69(2):282-286.

38. Chan E, Baumann O, Bellgrove MA, et al. Negative emotional experiences during navigation enhance parahippocampal activity during recall of place information. J Cogn Neurosci. 2014;26(1): 154-164.

39. Buhle JT, Kober H, Ochsner KN, et al. Common representation of pain and negative emotion in the midbrain periaqueductal gray. Soc Cogn Affect Neurosci. 2013;8(6):609-616.
40. Wiech K, Tracey I. The influence of negative emotions on pain: behavioral effects and neural mechanisms. Neuroimage. 2009;47(3):987-994.

41. Shuvaev VT, Shefer VI. Structure of neuronal activity of the caudate nucleus of monkeys during decision-making and the realization of the motor program in different variants of a delayed spatial choice task. Neurosci Behav Physiol. 1995;25(1):63-70.

42. Kesner RP, Gilbert PE. The role of the medial caudate nucleus, but not the hippocampus, in a matching-to sample task for a motor response. Eur J Neurosci. 2006;23(7):1888-1894.

43. Grahn JA, Parkinson JA, Owen AM. The cognitive functions of the caudate nucleus. Prog Neurobiol. 2008;86(3):141-155.

44. Yang J, Pan YJ, Zhao Y, et al. Oxytocin in the rat caudate nucleus influences pain modulation. Peptides. 2011;32(10):2104-2107.

45. Zhang Y, Yang C, Xu X, et al. Morphine dependence changes the role of droperidol on pain-related electric activities in caudate nucleus. Biochem Biophys Res Commun. 2008;372(1):179-185.
Neuropsychiatric Disease and Treatment

\section{Publish your work in this journal}

Neuropsychiatric Disease and Treatment is an international, peerreviewed journal of clinical therapeutics and pharmacology focusing on concise rapid reporting of clinical or pre-clinical studies on a range of neuropsychiatric and neurological disorders. This journal is indexed on PubMed Central, the 'PsycINFO' database and CAS,

\section{Dovepress}

and is the official journal of The International Neuropsychiatric Association (INA). The manuscript management system is completely online and includes a very quick and fair peer-review system, which is all easy to use. Visit http://www.dovepress.com/testimonials.php to read real quotes from published authors.

Submit your manuscript here: http://www.dovepress.com/neuropsychiatric-disease-and-treatment-journal 